\title{
Relación entre la calidad de los tratamientos de ortodoncia finalizados y la satisfacción en pacientes atendidos en la Universidad Santo Tomás desde 2018 hasta 2019
}

Relationship between the quality of orthodontic treatment and patient satisfaction in patients attended at Universidad Santo Tomás from 2018 to 2019

Relação entre a qualidade dos tratamentos ortodônticos concluídos e a satisfação dos pacientes atendidos na Universidad Santo Tomás de 2018 a 2019

\section{Ethman Ariel Torres ${ }^{1}$ Frank Nicolás Delgado-Morales ${ }^{2}$ Martha Juliana Rodríguez ${ }^{3}$}

Recibido: 26 de julio de 2020 Aprobado: 16 de diciembre de 2020 Publicado: 31 de diciembre de 2020

Cómo citar este artículo: Torres EA, Delgado-Morales FN, Rodríguez MJ. Relación entre la calidad de los tratamientos de ortodoncia finalizados y la satisfacción en pacientes atendidos en la Universidad Santo Tomás desde 2018 hasta 2019. Revista Nacional de Odontología. (2020); 16(2), 1-18. doi: https://doi.org/10.16925/2357-4607.2020.02.07

Artículo de investigación. https://doi.org/10.16925/2357-4607.2020.02.07

1 Facultad de Odontología, Universidad Santo Tomás; Bucaramanga, Colombia. Correo electrónico: ethman.torres@ustabuca.edu.co - ethmant@yahoo.com ORCID: https://orcid.org/0000-0003-1603-8380

2 Facultad de Ingeniería Industrial, Universidad Santo Tomás; Bucaramanga, Colombia.

3 Facultad de Odontología, Universidad Santo Tomás; Bucaramanga, Colombia. ORCID: https://orcid.org/0000-0001-7339-7836 
2 Relación entre la calidad de los tratamientos de ortodoncia finalizados y la satisfacción en pacientes atendidos en la Universidad Santo Tomás desde 2018 hasta 2019

\title{
Resumen
}

Introducción: el aumento en la prestación de los servicios de ortodoncia exige resultados de alta calidad para lograr la satisfacción del paciente, pero son escasos los estudios que abordan el tema.

Objetivo: determinar la relación entre la calidad de los tratamientos de ortodoncia finalizados con la satisfacción del paciente en las Clínicas del Ortodoncia de una universidad colombiana.

Métodos: se realizó un estudio observacional de corte transversal con una muestra de 67 pacientes que completaron su tratamiento de ortodoncia entre febrero de 2018 y junio de 2019. La satisfacción se evaluó con un cuestionario de 17 ítems. La calidad de los tratamientos de ortodoncia se analizó según los ocho criterios del Objective Grading System (OGS) de la American Board of Orthodontics sobre modelos de estudio y radiografías panorámicas tomadas al finalizar el tratamiento. Se calcularon frecuencias y proporciones para variables cualitativas y medidas de tendencia central y dispersión para las cuantitativas. Se utilizaron la prueba chi-cuadrado, exacta de Fisher y el coeficiente de correlación de Pearson según fuera apropiado. Un valor de $\mathrm{p}$ $<0,05$ se consideró estadísticamente significativo.

Resultados: entre los 67 participantes, 49 (73,1\%) eran mujeres, la edad promedio fue $21,4 \pm 9,1$ años, la duración promedio del tratamiento fue $32,3 \pm 5,0$ meses y el número promedio de defectos fue $28,8 \pm 9,2$. El $38.8 \%$ respondió que estaba muy satisfecho con los resultados. No se observó una relación entre la satisfacción del paciente y la calidad de los tratamientos de ortodoncia agrupados en excelentes, aceptables y deficientes de acuerdo con los criterios del OGS.

Conclusiones: no se encontró relación entre la calidad de los resultados del tratamiento de ortodoncia según los criterios del OGS y la satisfacción del paciente. Estos resultados mostraron que es necesario proponer planes de mejora continua en el centro universitario.

Palabras clave: ortodoncia, satisfacción del paciente, normas, calidad de la atención en salud [DeCS]

\begin{abstract}
Introduction: The increasing orthodontic service demands high-quality outcomes so the patient could be satisfied with the results, but scarce studies are addressing this topic.

Objective: To assess the relationship between the quality of orthodontic treatment outcomes and patient satisfaction at an Orthodontic Clinic in a Colombian university.

Methods: An observational cross-sectional study was conducted with a sample of 67 patients who completed their orthodontic treatment between February 2018 and June 2019. Satisfaction was assessed by a questionnaire consisting of 17 items. The quality of the orthodontic treatments was established with post-treatment study models and panoramic radiographs that were analyzed according to the eight criteria of the Objective Grading System (OGS) generated by the American Board of Orthodontics. Frequencies and proportions for qualitative variables and measures of central tendency and dispersion for quantitative ones were calculated. Chi-square test, Fisher exact test, and Pearson correlation coefficients were used as appropriate. $P<0.05$ was considered statistically significant.

Results: Among the 67 participants, 49 (73.1\%) were female, the average age was $21.4 \pm 9.1$ years, the average treatment duration was $32.3 \pm 5.0$ months, and the average defects was $28.8 \pm 9.2$. For the overall satisfaction score, $38.8 \%$ were very satisfied. There was not a relationship between patient satisfaction and the quality of the orthodontics treatments grouped in excellent, acceptable, and deficient according to the OGS criteria.

Conclusions: No relationship was found between the quality of the orthodontic treatment outcomes measured by OGS criteria and patient satisfaction. These results showed that it is necessary to propose continuous improvement plans at the university center.
\end{abstract}

Key words: orthodontics, patient satisfaction, standards, quality of health care [MeSH] 


\section{Resumo}

Introdução: O crescente serviço ortodôntico exige resultados de alta qualidade para que o paciente possa ficar satisfeito com os resultados, mas estudos escassos abordam esse tópico.

Objetivo: Avaliar a relação entre a qualidade dos resultados do tratamento ortodôntico e a satisfação do paciente em uma clínica ortodôntica em uma universidade colombiana.

Métodos: Foi realizado um estudo observacional transversal com uma amostra de 67 pacientes que completaram o tratamento ortodôntico entre fevereiro de 2018 e junho de 2019. A satisfação foi avaliada por um questionário composto por 17 itens. A qualidade dos tratamentos ortodônticos foi estabelecida com modelos de estudo pós-tratamento e radiografias panorâmicas que foram analisadas de acordo com os oito critérios do Objective Grading System (OGS) gerado pelo American Board of Orthodontics. Foram calculadas frequências e proporções para variáveis qualitativas e medidas de tendência central e dispersão para quantitativas. 0 teste do qui-quadrado, o teste exato de Fisher e os coeficientes de correlação de Pearson foram utilizados conforme apropriado. $\mathrm{P}<0,05$ foi considerado estatisticamente significante.

Resultados: Dos 67 participantes, 49 (73,1\%) eram do sexo feminino, a idade média foi de 21,4 $\pm 9,1$ anos, a duração média do tratamento foi de $32,3 \pm 5,0$ meses e os defeitos médios foram de $28,8 \pm 9,2$. Para o índice de satisfação geral, 38,8\% ficaram muito satisfeitos. Não houve relação entre a satisfação do paciente e a qualidade dos tratamentos ortodônticos, agrupados em excelente, aceitável e deficiente de acordo com os critérios da OGS.

Conclusões: Não foi encontrada relação entre a qualidade dos resultados do tratamento ortodôntico medidos pelos critérios da OGS e a satisfação do paciente. Esses resultados mostraram que é necessário propor planos de melhoria contínua no centro universitário.

Palavras-chave: ortodontia, satisfação do paciente, normas, qualidade da assistência à saúde [DeCS]

\section{Introducción}

Mejorar la calidad de los tratamientos y alcanzar una alta satisfacción de los pacientes se ha convertido en una meta esencial en los centros de atención en salud, lo que promueve la búsqueda de altos estándares de calidad y competitividad que permitan la supervivencia en el mercado (1). Es así como en el campo de la ortodoncia, se han reportado algunos estudios recientes sobre la calidad de los tratamientos finalizados con la satisfacción de los pacientes $(2,3)$.

Al tratar el tema sobre la calidad de tratamientos de ortodoncia finalizados, los parámetros del Objetive Grading System (OGS) del American Board of Orthodontic (ABO) propuestos por Casko et al. en 1998 (4). Estos criterios examinan ocho variables en modelos de estudio y radiografías panorámicas para determinar el número de defectos que presenta el caso finalizado, un caso con menos de veinte defectos se considera como "excelente o aprobado" y con más de treinta defectos como "deficiente sin aprobación" (4). Este OGS es un instrumento replicado y utilizado por diferentes universidades del mundo como lo demuestra la revisión sistemática y meta-análisis publicada por Papageorgiou et al. en 2017 (5), que describe 34 estudios que han 
4 Relación entre la calidad de los tratamientos de ortodoncia finalizados y la satisfacción en pacientes atendidos en la Universidad Santo Tomás desde 2018 hasta 2019

utilizado esta metodología de evaluación de calidad. Si bien se ha mostrado la utilidad del instrumento para mejorar la calidad, también se han reportado deficiencias. Chung et al. (6) reportan que los errores más comunes son los detalles de finalización, los objetivos de tratamiento inadecuados, la excesiva inclinación de incisivos inferiores, el aumento excesivo del diámetro intercanino, el bajo control de la dimensión vertical y las fallas en el trazo cefalometrico.

El objetivo de realizar este seguimiento de calidad en los tratamientos finalizados de ortodoncia esta alineado con estándares internacionales que deben fomentar los programas de especialización en ortodoncia como el propuesto por Allareddy et al. en 2019 (7), en donde los parámetros adecuados de finalización y el seguimiento de calidad de los tratamientos son factores muy importantes que indican la excelencia de cada programa. Con la utilización adecuada del OGS y haciendo de este instrumento una herramienta en planes de mejora continua, los programas de formación en ortodoncia han mostrado que la implementación de protocolos y guias de finalización mejoran los niveles de calidad de los tratamientos; un ejemplo de ello, lo reporta Barbosa-Lis et al. en 2014 (8), donde luego de implementar un protocolo de finalización se observaron mejoras en la calidad de los tratamientos según los criterios del OGS (9). De igual manera, Verma et al. (10) implementaron un protocolo de finalización basados en el seguimiento según los criterios del OGS y lograron mejorar los resultados de calidad.

Al revisar estudios sobre la satisfacción de los pacientes después de recibir el tratamiento de ortodoncia se encontró la revisión de Pacheco-Pereira et al. (11) quienes reportan que dos de los factores más frecuentemente asociados con la satisfacción fueron el trato personal y la relación entre el profesional y el paciente; en tanto que aspectos relacionados con la estética y la estabilidad de los resultados son los que más inciden en la baja satisfacción luego de finalizar un tratamiento de ortodoncia.

La evaluación de la satisfacción se ha realizado a través de cuestionarios diseñados para tal fin. Alfaro et al. (12) indagaron sobre la satisfacción del paciente luego de haber terminado su tratamiento de ortodoncia en una universidad comparado con cuatro consultorios privados. Aplicaron un cuestionario con 52 preguntas y encontraron una diferencia estadísticamente significativa $(p=0,0361)$ en la dimensión "satisfacción con los aspectos circunstanciales" que se refería a la comodidad de la sala de espera y de las instalaciones, la duración del tratamiento y el costo de este. De otra parte, Agudelo et al. en 2008 (13) presentaron un instrumento basado en ocho dimensiones que evaluó la percepción del usuario respecto a la atención recibida en una Institución Prestadora de Servicios de Salud (IPS). Este cuestionario luego fue aplicado por Torres-Gonzáles y León-Manco (14) en pacientes que habían finalizado 
su tratamiento en un programa de posgrado en ortodoncia de una clínica dental peruana. Una de las ventajas de este instrumento es la evaluación de variables propias de centros de formación docente asistencial.

Si bien la evaluación de la calidad de los tratamientos de ortodoncia finalizados y la satisfacción de los pacientes se han estudiado en diferentes centros de formación de ortodoncistas, aún no se ha reportado un estudio que analice la relación entre estos dos aspectos. Por este motivo, el objetivo del presente trabajo fue determinar la relación entre la calidad de los tratamientos de ortodoncia finalizados según los criterios del OGS de la ABO con la satisfacción del paciente en las Clínicas del Ortodoncia de una universidad colombiana.

\section{Materiales y métodos}

Se realizó un estudio observacional descriptivo de corte transversal cuya población estuvo conformada por 87 pacientes que habían finalizado su tratamiento de ortodoncia en las Clínicas de Ortodoncia de la Universidad Santo Tomas; seccional Bucaramanga; en el periodo comprendido entre febrero de 2018 y junio de 2019. Este número de pacientes no incluía a los que habían sido sometidos a cirugía maxilofacial o rehabilitación oral.

Se obtuvo un tamaño de muestra de 72 pacientes a partir de las siguientes especificaciones: una población de 87 pacientes, un nivel de confianza del 95\%, una prevalencia de satisfacción con el tratamiento del 50\% y un efecto del diseño de 1 (15). Se adicionó un 10\% de proporción de no respuesta para un tamaño de muestra definitivo de 79 pacientes.

Se realizó un muestreo probabilístico aleatorio y se incluyeron los pacientes que habían terminado su tratamiento de ortodoncia un un periodo máximo de tres meses a partir del contacto. A cada uno se le tomó una radiografía panorámica, modelos de estudio, y se realizó una entrevista telefónica para indagar sobre la satisfacción con el tratamiento de ortodoncia. 12 pacientes no se pudieron contactar para la entrevista o no asistieron a la toma de radiografia de control antes de los tres meses de finalizado su tratamiento, por lo que la poblacion de estudio estuvo conformada por 67 pacientes.

Se incluyeron variables sociodemográficas (edad y sexo), duración del tratamiento de ortodoncia en meses según la historia clínica, criterios del OGS (alineación, rebordes marginales, inclinación bucolingual, contactos oclusales, relación oclusal, overjet, contactos interpoximales y angulación de raíz), criterios de calidad de la 
American Board of Orthodontics (ABO) y las relacionadas con el cuestionario sobre satisfacción con el tratamiento de ortodoncia.

\section{Instrumento para evaluar la satisfacción con el tratamiento}

Se utilizó el cuestionario de satisfacción creado por Agudelo et al. (13) en la Universidad de Antioquía. El instrumento 17 ítems agrupados en ocho dimensiones que son: 1) trato personal, 2) eficacia en la atención y satisfacción, 3) información recibida, 4) accesibilidad, 5) oportunidad, 6) seguridad y privacidad, 7) instalaciones, equipos y materiales, 8) satisfaccion general. En lo referente a satisfacción, se preguntó por la satisfacción con los resultados estéticos logrados y con la oclusión o "mordida" alcanzada. La opciones de respuesta se distribuyen en una escala Likert que se codifican como 1="muy insatisfecho", 2="insatisfecho", 3="indiferente", 4="satisfecho" y 5="muy satisfecho".

La confiabilidad del cuestionario se evaluó al estimar su consistencia interna con el alpha de Cronbach, se obtuvo un coeficiente de 0,96 que es considerado "excelente" según la interpretación de Fayers y Machin (16). Para evaluar la validez del cuestionario, se utilizó el método normalizado de Tristán-López (2008), cuyo resultado fue "bueno" (17).

\section{Evaluación de la calidad de los modelos}

Se realizó un análisis estático de los modelos y de la radiografía panorámica aplicando los criterios del Objective Grading System (OGS): alineación, rebordes marginales, inclinación buco-lingual, relaciones oclusales, contactos oclusales, sobre mordida horizontal (overjet), contactos interproximales y angulación de la raíz. Para medir este puntaje se utilizo el "ABO measuring gauge" y los lineamientos de la ABO.

Los resultados finales del análisis de modelos y de las radiografías panorámicas se agruparon siguiendo la guía del OGS: los puntajes iguales o menores a 20 se consideraron excelentes; entre 21 y 30 se consideraron aceptables y puntajes superiores a 30 , menos que aceptables (4).

Previo al análisis de los modelos de estudio, se realizó una calibración interexaminador y se calculó el Coeficiente Kappa de Cohen que fue interpretado según la escala que presentada por Landis y Koch en 1977 (Tabla 1) (18). 
Tabla 1. Calibración interexaminador de los modelos de estudio.

\begin{tabular}{lcl}
\hline \multicolumn{1}{c}{ Característica } & Coeficiente Kappa & Interpretación \\
\hline Alineación & 0,70 & Considerable \\
\hline Rebordes marginales & 0,84 & Casi perfecta \\
\hline Inclinación bucolingual & 0,93 & Casi perfecta \\
\hline Contactos oclusales & 0,70 & Considerable \\
\hline Relación oclusal & 1,00 & Casi perfecta \\
\hline Overjet & 0,93 & Casi perfecta \\
\hline Contactos interproximales & 1,00 & Casi perfecta \\
\hline Angulación de la raíz & 0,90 & Casi perfecta \\
\hline
\end{tabular}

Fuente: elaboración propia

Los datos fueron registrados en dos bases de datos creadas para tal fin. Estas fueron validadas con el software Epidata 3,1 y los errores de digitación fueron corregidos con los instrumentos. Una base completamente depurada fue exportada al programa estadístico Stata I/C versión 14. La variable duración del tratamiento fue categorizada con un punto de corte en 28 meses según las recomendaciones de Papageorgiou et al. (5).

\section{Análisis estadístico}

Se realizó un análisis descriptivo en el que se calcularon frecuencias y proporciones para las variables cualitativas, y medidas de tendencia central y dispersión para las cuantitativas. En el análisis bivariado, se utilizó el test Exacto de Fisher para las variables cualitativas y el coeficiente de correlación de Pearson para las variables cuantitativas. Un valor de $p<0,05$ fue considerado como estadísticamente significativo.

\section{Consideraciones éticas}

Este trabajo fue aprobado por el Comité de Ética de la Facultad de Odontología de la Universidad Santo Tomás según Acta del 24 de mayo de 2018. Según la Resolución 008430 del 4 octubre 1993, se clasificó como una "investigación con riesgo mayor al mínimo" debido a que se tomaron radiografías panorámicas de los pacientes (19). Se realizó el proceso de Consentimiento Informado en el que se explicó a cada paciente el objetivo y el procedimiento para llevar a cabo el estudio. Se respetaron los principios de autonomía, justicia, no maleficencia y beneficencia. 
8 Relación entre la calidad de los tratamientos de ortodoncia finalizados y la satisfacción en pacientes atendidos en la Universidad Santo Tomás desde 2018 hasta 2019

\section{Resultados}

La población de estudio la constituyeron 67 pacientes lo que arrojó una proporción de respuesta del 93,1\%. Se evaluaron las radiografías y los modelos de 49 (73,1\%) mujeres, el promedio de la edad de los 67 participantes fue 21,4 \pm 9,1 años (Mediana: 19, Rango Intercuartílico: 16 - 23 años). El promedio de duración del tratamiento de ortodoncia fue 32,3 \pm 5,0 meses [IC 95\% 31,1 - 33,5].

\section{Evaluación de la calidad en los modelos de estudios según el OGS}

Se obtuvo un promedio de 28,8 \pm 9,2 puntos [IC 95\% 26,6 - 31,0] que según la clasificación sugerida por la ABO correspondería a doce $(17,9 \%)$ casos de calificación "excelente", 31 (46,3\%) casos designados como "aceptable", y 24 (35,8\%) casos como "deficiente". Al integrar los casos "excelentes" con los "aceptables", se obtendrían 43 $(64,2 \%)$ casos que cumplían los criterios de la ABO, con menos de treinta puntos. De esta manera, 24 (35,8\%) casos no cumplían con los criterios mínimos al finalizar su tratamiento.

La Tabla 2 muestra los resultados obtenidos en los 67 modelos según las variables de la OGS. Se observa la proporción de defectos encontrados por diente analizado; se encontró una proporción mayor de defectos en la zona de molares según las variables alineación, rebordes marginales e inclinación. Así mismo, se evidenció una alta proporción de defectos en dientes anteriores según las variables overjet y angulación de raíz.

Tabla 2. Proporción de defectos en cada uno de los dientes analizados en modelos de pacientes con tratamiento de ortodoncia finalizado en las Clínicas de Ortodoncia de la Universidad Santo Tomás.

\begin{tabular}{|c|c|c|c|c|c|c|c|c|c|c|c|c|c|c|}
\hline \multirow{2}{*}{ Variable } & \multicolumn{7}{|c|}{ Lado Derecho (\%) } & \multicolumn{7}{|c|}{ Lado Izquierdo (\%) } \\
\hline & 7 & 6 & 5 & 4 & 3 & 2 & 1 & 1 & 2 & 3 & 4 & 5 & 6 & 7 \\
\hline \multicolumn{15}{|l|}{ Alineación } \\
\hline Superior & 25,3 & 26,8 & 8,9 & 4,4 & 8,9 & 20,9 & 0,0 & 1,5 & 5,9 & 7,5 & 13,4 & 16,4 & 28,3 & 28,3 \\
\hline Inferior & 37,3 & 32,8 & 8,9 & 8,9 & 2,9 & 2,9 & 0,0 & 1,5 & 8,9 & 5,9 & 11,9 & 8,5 & 23,8 & 28,3 \\
\hline \multicolumn{15}{|l|}{ Rebordes } \\
\hline Superior & 46.3 & 41.8 & 22.4 & 4.4 & - & & & & & & 22.4 & 28.3 & 29.8 & 54.8 \\
\hline Inferior & 71.4 & 40.3 & 25.3 & 1.5 & - & - & - & - & - & - & 1.5 & 17.9 & 50.3 & 70.2 \\
\hline
\end{tabular}


(viene)

\begin{tabular}{|c|c|c|c|c|c|c|c|c|c|c|c|c|c|c|}
\hline \multirow{2}{*}{ Variable } & \multicolumn{7}{|c|}{ Lado Derecho (\%) } & \multicolumn{7}{|c|}{ Lado Izquierdo (\%) } \\
\hline & 7 & 6 & 5 & 4 & 3 & 2 & 1 & 1 & 2 & 3 & 4 & 5 & 6 & 7 \\
\hline \multicolumn{15}{|l|}{ Inclinación } \\
\hline Superior & 70,2 & 11,9 & 4,5 & 5,9 & - & - & - & - & - & - & 8,9 & 2,9 & 23,8 & 65,7 \\
\hline Inferior & 62,7 & 26,8 & 28,4 & 22,4 & - & & & & & & 28,4 & 32,8 & 17,9 & 59,7 \\
\hline \multicolumn{15}{|l|}{ Contactos } \\
\hline Superior & 23,8 & 4,4 & 25,4 & 10,5 & - & - & - & - & - & - & 2,9 & 28,4 & 7,4 & 14,9 \\
\hline Inferior & 22,4 & 4,4 & 2,9 & 2,9 & - & & & & & - & 2,9 & 0,0 & 5,9 & 11,9 \\
\hline \multicolumn{15}{|l|}{ Overjet } \\
\hline Superior & 14,9 & 7,4 & 4,4 & 7,4 & 32,8 & 19,5 & 8,9 & 5,9 & 16,4 & 23,8 & 0,0 & 6,4 & 11,9 & 14,9 \\
\hline Inferior & 13,4 & 8,9 & 1,5 & 0,0 & 25,4 & 13,4 & 4,4 & 5,9 & 13,4 & 17,9 & 1,5 & 1,5 & 4,4 & 5,9 \\
\hline \multicolumn{15}{|l|}{ Espacios } \\
\hline Superior & 0,0 & 0,0 & 2,9 & 0,0 & 1,5 & 0,0 & 0,0 & 0,0 & 5,9 & 10,4 & 0,0 & 0,0 & 0,0 & 0,0 \\
\hline Inferior & 0,0 & 0,0 & 10,4 & 0,0 & 7,4 & 2,9 & 0,0 & 0,0 & 0,0 & 8,9 & 0,0 & 7,4 & 0,0 & 1,5 \\
\hline \multicolumn{15}{|c|}{ Angulación raíz } \\
\hline Superior & 0,0 & 0,0 & 26,8 & 5,7 & 25,3 & 50,3 & 2,9 & 2,9 & 52,4 & 19,4 & 2,9 & 31,4 & 0,0 & 1,4 \\
\hline Inferior & 0,0 & 0,0 & 16,4 & 22,4 & 31,3 & 25,4 & 7,4 & 7,4 & 20,8 & 40,3 & 21,3 & 10,4 & 0,0 & 0,0 \\
\hline
\end{tabular}

1: Incisivo central. 2: Incisivo lateral. 3: Canino. 4: Primer premolar. 5: Segundo premolar. 6: Primer molar.

7: Segundo molar.

Fuente: elaboración propia

\section{Satisfacción de los pacientes luego de concluir el tratamiento ortodóntico}

Al evaluar el puntaje total del cuestionario, se evidenció que $26(38,8 \%)$ pacientes respondieron "muy satisfechos" en todos los ítems del cuestionario. El promedio de los puntajes fue 77,1 \pm 10,5 (Me: 82 y RIC 72 - 85). Ningún ítem fue registrado como "muy insatisfecho".

Se observó que 50 (74,6\%) pacientes se encontraban "Muy satisfechos" con el trato personal recibido. En tanto que el ítem sobre accesibilidad y oportunidad relacionado con la asignación de citas obtuvo la mayor proporción de "insatisfechos" (Tabla 3). 
Tabla 3. Distribución de frecuencias de la percepción de satisfacción con el servicio de ortodoncia en las Clínicas de Ortodoncia de la Universidad Santo Tomás.

\begin{tabular}{lcccc}
\hline \multicolumn{1}{c}{ Variable } & $\begin{array}{c}\text { Insatisfecho } \\
\mathbf{n}(\mathbf{\%})\end{array}$ & $\begin{array}{c}\text { Indiferente } \\
\mathbf{n}(\mathbf{\%})\end{array}$ & $\begin{array}{c}\text { Satisfecho } \\
\mathbf{n}(\mathbf{\%})\end{array}$ & $\begin{array}{c}\text { Muy satisfecho } \\
\mathbf{n}(\mathbf{\%})\end{array}$ \\
\hline Satisfacción en el trato personal & $1(1,5)$ & $2(3,0)$ & $14(20,9)$ & $50(74,6)$ \\
\hline Satisfacción con el servicio & $2(3,0)$ & $9(13,4)$ & $15(22,4)$ & $41(61,2)$ \\
\hline $\begin{array}{l}\text { Satisfacción con los resultados } \\
\text { estéticos }\end{array}$ & $3(4,5)$ & $8(11,9)$ & $15(22,4)$ & $41(61,2)$ \\
\hline $\begin{array}{l}\text { Satisfacción con los resultados } \\
\text { en oclusión }\end{array}$ & $5(7,5)$ & $8(11,9)$ & $17(25,4)$ & $37(55,2)$ \\
\hline $\begin{array}{l}\text { Satisfacción con la información } \\
\text { suministrada }\end{array}$ & $2(3,0)$ & $5(7,5)$ & $15(22,3)$ & $45(67,2)$ \\
\hline $\begin{array}{l}\text { Satisfacción con la accesibilidad } \\
\text { y oportunidad }\end{array}$ & $6(9,0)$ & $13(19,4)$ & $13(19,4)$ & $35(52,2)$ \\
\hline $\begin{array}{l}\text { Satisfacción con la seguridady } \\
\text { privacidad en la consulta }\end{array}$ & $1(1,5)$ & $6(9,0)$ & $11(16,4)$ & $47(70,2)$ \\
\hline $\begin{array}{l}\text { Satisfacción con las instalacio- } \\
\text { nes, equipos y materiales }\end{array}$ & $1(1,5)$ & $4(6,0)$ & $15(22,3)$ & $47(70,2)$ \\
\hline \begin{tabular}{l} 
Satisfacción general \\
\hline
\end{tabular} & $2(3,0)$ & $4(6,0)$ & $18(26,9)$ & $43(64,1)$ \\
\hline
\end{tabular}

Fuente: elaboración propia

Al analizar el sexo, la edad y la duración del tratamiento con la calidad del tratamiento agrupado según las indicaciones del OGS, se encontró una diferencia estadísticamente significativa en la calidad del tratamiento según el sexo $(p=0,010)$ dado que más de la mitad de las mujeres participantes señalaron como "aceptable" la calidad del tratamiento (Tabla 4). Al relacionar el tiempo de duración del tratamiento con el puntaje global del cuestionario sobre satisfacción, se observó una relación despreciable y negativa que no fue estadísticamente significativa (pearson: -0,1165 $p=0,3478)$.

Tabla 4. Relación entre el sexo, la edad y la duración del tratamiento con la calidad del tratamiento según el OGS.

\begin{tabular}{lcccc}
\hline \multirow{2}{*}{ Variable } & \multicolumn{3}{c}{ Calidad del Tratamiento } & P \\
\cline { 2 - 4 } & Excelente $\mathbf{n ~ ( \% )}$ & Aceptable $\mathbf{n ~ ( \% ) ~}$ & Deficiente $\mathbf{n ~ ( \% ) ~}$ & \\
\hline Sexo & & & & 0,010 \\
\hline Femenino & $5(10,2)$ & $27(55,1)$ & $17(34,7)$ & \\
\hline Masculino & $7(38,9)$ & $4(22,2)$ & $7(38,9)$ & 0,250 \\
\hline Edad (años) & & & & $15(38,5)$ \\
\hline Menor a 20 & $9(23,1)$ & $15(38,5)$ & $9(32,1)$ & \\
\hline Igual o mayor a 20 & $3(10,7)$ & $16(57,1)$ & & \\
\hline
\end{tabular}


(viene)

\begin{tabular}{lcccc}
\hline \multirow{2}{*}{ Variable } & \multicolumn{3}{c}{ Calidad del Tratamiento } & \multirow{2}{*}{ P } \\
\cline { 2 - 4 } & Excelente $\mathbf{n}(\%)$ & Aceptable $\mathbf{n}(\%)$ & Deficiente $\mathbf{n}(\%)$ & \\
\hline Duración del tratamiento (meses) & & $4(25,0)$ & \\
\hline Menor o igual 28 & $5(31,2)$ & $7(43,8)$ & $20(39,2)$ \\
\hline Mayor a 28 & $7(13,7)$ & $24(47,1)$ & \\
\hline
\end{tabular}

Test Exacto de Fisher. OGS: Objective Grading System.

Fuente: elaboración propia

En la Tabla 5 se observa la relación entre la calidad del tratamiento según los criterios de la ABO y la percepción de satisfacción reportada por el participante, no se encontró una diferencia estadísticamente significativa entre las variables evaluadas. No obstante, es importante señalar que una mayor proporción de pacientes reportó como "aceptable" la calidad del tratamiento.

Tabla 5. Relación entre la satisfacción del paciente y la calidad del tratamiento según $A B O$.

\begin{tabular}{|c|c|c|c|c|}
\hline \multirow{2}{*}{ Variable } & \multicolumn{3}{|c|}{ Calidad del Tratamiento } & \multirow{2}{*}{$\mathbf{P}$} \\
\hline & Excelente n (\%) & Aceptable n (\%) & Deficiente n (\%) & \\
\hline Satisfacción general & & & & 0,469 \\
\hline No satisfecho & $2(33,3)$ & $2(33,3)$ & $2(33,3)$ & \\
\hline Satisfecho & $10(16,4)$ & $29(47,6)$ & $22(36,1)$ & \\
\hline Satisfacción con la estética & & & & 0,189 \\
\hline No satisfecho & $4(36,4)$ & $3(27,2)$ & $4(36,4)$ & \\
\hline Satisfecho & $8(14,3)$ & $28(50,0)$ & $20(35,7)$ & \\
\hline Satisfacción con oclusión & & & & 0,084 \\
\hline No satisfecho & $4(40,0)$ & $2(20,0)$ & $4(40,0)$ & \\
\hline Satisfecho & $8(14,0)$ & $29(50,9)$ & $20(35,1)$ & \\
\hline
\end{tabular}

Test Exacto de Fisher. ABO: American Board of Orthodontics.

Fuente: elaboración propia

\section{Discusión}

Al evaluar la calidad del tratamiento en 67 pacientes con tratamientos ortodoncia finalizados y su relación con la satisfacción del paciente, no se encontró una relación entre las medidas tomadas en modelos y radiografías al tener en cuenta los criterios 
sugeridos por la ABO y la satisfacción expresada por los pacientes mediante un cuestionario. Si bien los resultados de calidad no alcanzan los niveles sugeridos en la clasificación del OGS, los pacientes expresaron quedar satisfechos con sus tratamientos y esto se evidenció en los resultados obtenidos. A continuación, se discutirán los hallazgos de forma detallada.

\section{Calidad de modelos según el OGS de la ABO}

El estudio encontró un promedio del OGS de 28,8 \pm 9,2 puntos [IC 95\% 26,6 - 31,0] lo que estaría dentro del promedio mostrado por la revisión de Papageorgiou et al. en 2017 porque en 34 estudios realizados con 6207 pacientes encontró un OGS de 27,9 [IC 95\% 25,3 - 30,6] (5). En cuanto a la distribución según la proporción del puntaje encontrado en el presente estudio, solo el $64,2 \%$ de la muestra cumplía con los criterios de la $\mathrm{ABO}$ al tener menos de treinta puntos, mientras el 35,8\% se consideró como deficiente al tener más de 30 puntos, se considera como deficiente (4).

Resultados similares en distribución de los casos según el puntaje OGS fueron reportados por Santiago y Martínez quienes, de un total de 64 casos evaluados, encontraron un promedio del OGS de 32,2 \pm 13,0 puntos de los cuales un 18,8\% fueron considerados como excelentes, $34,4 \%$ se consideraban como aceptables y el $46,9 \%$ de la muestra no cumplía con los criterios exigidos por la ABO (20). También se encuentran reportes de OGS con mejores resultados como el de Cansunar y Uysal quienes evaluaron 1693 casos y reportaron un OGS de 18,0 (21). Aunque la ABO considera como excelente todo caso con menos de veinte puntos, en algunas universidades como la de Pekín, Song et al. establecen como criterio que todo caso debe tener menos de 16 puntos; un caso con más de 21 puntos es considerado como deficiente e inaceptable según la estandarización de sus guías de manejo (22). Esta evidencia sugiere que cada centro de educación debe estandarizar y controlar el proceso de finalización de sus casos con el fin de aumentar la rigurosidad en la exigencia de la calidad.

Al comparar los resultados obtenidos en este trabajo con otros estudios similares realizados en los programas de posgrado de ortodoncia en Colombia, se encontró que Barbosa et al. (8) en muestra de cuarenta pacientes registraron un OGS de 31,7 $\pm 8,5$ puntos y luego de aplicar un protocolo de finalización mejoraron los resultados obtenidos (9). De otra parte, en la Universidad Autónoma de Manizales Barrera et al. (23) encontraron un OGS de 30,9 \pm 11,0 puntos en una muestra de 31 pacientes.

En esta investigación no se tuvo en cuenta variables como el índice de maloclusion que tenia el paciente al inicio del tratamiento, la técnica ortodontica empleada y 
la realización o no de exodoncias, por lo que no se relacionaron estas variables con el resultado final del OGS. Estas variables son tomadas en cuenta en otras publicaciones sobre el OGS de la ABO, como la de Campbell et al. (24) en la Universidad de Indiana quienes reportan mayores problemas como resultado en los casos de mayor complejidad, por lo que recomienda un seguimiento y control especial en ellos. Yang-Powers et al. (25) de la Universidad de Illinois consideran que las fallas en casos de mayor complejidad se relacionan con el torque en molares y paralelismo de raíces por lo que también muestran relación entre grado inicial de la maloclusion y el la calidad del resultado al finalizar el tratamiento. Estos trabajos recomiendan que en estos casos, el docente debe llevar un seguimiento y un control más estricto.

\section{Duración del tratamiento}

En cuanto a duración del tratamiento, la revisión de Papageorgiou et al. (26) muestra un valor promedio de duración del tratamiento de ortodoncia para los 34 estudios analizados de 24,9 meses (IC 95\% 24,6 - 25,1). En el presente estudio, la duración promedio del tratamiento de los 67 casos es superior al ser 32,3 meses (IC 95\% 31,1 - 33,5). El rango de tratamiento mostrado en la revisión es amplio porque informa desde tratamientos con duración de 19 meses hasta tratamientos de 56 meses y sugiere que el la duración esta directamente relacionada con la complejidad de la maloclusion, aunque no reporta algún estudio que demuestre de forma evidente esta relación. En la presente investigación al relacionar la duración del tratamiento con la calidad del mismo según el OGS se encontró un valor de $p=0,246$ lo que no muestra una relación entre las variables.

De igual modo, la revisión sistemática de Tsichlaki et al. (27) señala cuánto debe durar un tratamiento de ortodoncia con aparatología fija y menciona que el promedio de duración para los 22 estudios incluidos en 1089 participantes fue de 19,9 meses [IC 95\% 19,6 - 20,2]. Al ser mayor la duración del tratamiento de ortodoncia hallada en este trabajo, se considera que es factor a tener en cuenta para establecer un mejor seguimiento y control. Sin embargo, es importante señalar que al ser los tratamientos finalizados en una institución de educación superior, los periodos de vacaciones y la transferencia de pacientes pueden alargar la duración de los tratamientos.

\section{Satisfacción del paciente}

En este trabajo, se observó que el valor de satisfacción más alto fue en el ítem sobre el trato personal y el de más baja satisfacción se relacionó con la accesibilidad 
y la oportunidad. Al evaluar la satisfacción general, se encontró que el 64,2\% de los pacientes se encontraba "satisfecho". Agudelo et al. (13), también encontró una alta satisfacción global $(95,9 \%)$ resaltando la confianza técnica y profesional del personal de la institución como factor importante en el logro de ese resultado, también sugiere que algunos factores a mejorar se relacionan con la privacidad y la oportunidad. De otra parte, Torres-Gonzáles y León-Manco (14) reportaron un alto nivel de satisfacción global $(84,0 \%)$ en los pacientes atendidos en el servicio de ortodoncia.

Al revisar los resultados del presente estudio en cuanto a satisfacción con la revisión sistemática de Pacheco-Pereira et al. (11) en la que se analizaron 18 estudios con 2891 pacientes, se destaca que uno de los factores más relacionados con la satisfacción del paciente es la percepción subjetiva que incluye el trato personal y la relación entre el paciente, el profesional y su equipo de trabajo. En este trabajo este aspecto obtuvo el mayor porcentaje de satisfacción, como ya se ha mencionado.

Los factores objetivos relacionados con estética y oclusión, también se han reportado como importantes en el momento de determinar el grado de satisfacción. en este estudio se reporto un 61,2\% y 59,7\% de "muy satisfechos", respectivamente. Sin embargo, cuando estos factores se relacionaron con la calidad de los modelos según los criterios del OGS, no se encontró una relación. La revisión también reportó que los factores negativos más relacionados con la baja satisfacción del paciente se relacionan con episodios de dolor o incomodidades durante el tratamiento, al igual que los problemas referidos con la retención y estabilidad; estos factores no fueron evaluado en este trabajo ya que el cuestionario se aplicó a un máximo de tres meses luego de retirar los aparatos ortodónticos, y en este periodo de tiempo no es frecuente observar alteraciones con la estabilidad de los tratamientos. También se ha reportado baja satisfacción con el tiempo de duración del tratamiento; en este estudio se presentó una mayor duración del tratamiento pero esta situación no se relacionó con la satisfacción al hallarse una correlación despreciable [pearson: -0,1165 p=0,3478].

\section{Limitaciones}

Una de las limitaciones de este trabajo fue no relacionar la calidad de los modelos del tratamiento terminado con el índice inicial de la maloclusión, la técnica de ortodoncia utilizada y la realización de exodoncias. La razón para ello fue la falta en la estandarización de estos datos a partir de las historias clínicas. No obstante, no fueron tenidos en cuenta aquellos pacientes sometidos a procedimientos de cirugía maxilofacial y rehabilitación oral que estarían relacionados con los casos de mayor complejidad. De manera rutinaria no es utilizado este instrumento de medicion por parte de los 
docentes y estudiantes del postgrado; lo que dificulta el proceso de calibracion y realizar un seguimiento de los resultados.

\section{Conclusiones}

No se encontró una relación entre la calidad de los modelos según el OGS de la ABO y la satisfacción del paciente luego de finalizado su tratamiento de ortodoncia. Así mismo, el promedio de calidad según la OGS fue 28,8 \pm 9,2 puntos, donde el 35,8\% de la muestra no cumplió con los criterios mínimos exigidos por la ABO.

Este estudio es un punto de partida para el desarrollo de un plan de mejoramiento institucional que incluye a la comunidad administrativa, docente y estudiantil con el fin de elaborar y adoptar guías clínicas de finalización de los tratamientos de ortodoncia en las clínicas odontológicas de la universidad, y de esta manera, lograr unos altos estándares en el tratamiento integral de los pacientes.

\section{Conflicto de intereses}

Los autores declaran que no existe ningún conflicto de interés

\section{Financiamiento}

Este trabajo fue financiado en la X Convocatoria Interna de Investigación de la Universidad Santo Tomás Seccional Bucaramanga según consta en el Acta 0442 de 2017 del Comité Administrativo-Financiero.

\section{Referencias}

1. Porter ME, Teisberg EO. Redefining competition in health care. Harv Bus Rev. 2004;82(6):6476,136

2. Lee R, Hwang S, Lim H, Cha JY, Kim KH, Chung CJ. Treatment satisfaction and its influencing factors among adult orthodontic patients. Am J Orthod Dentofacial Orthop. 2018;153(6):80817. DOI: 10.1016/j.ajodo.2017.09.015 
Relación entre la calidad de los tratamientos de ortodoncia finalizados y la satisfacción en pacientes atendidos en la Universidad Santo Tomás desde 2018 hasta 2019

3. Utari E, Ati K, Ismah N. Relationship between orthodontic treatment outcome and patient satisfaction. Journal of International Dental and Medical Research. 2017;10:503-14.

4. Casko JS, Vaden JL, Kokich VG, Damone J, James RD, Cangialosi TJ, et al. Objective grading system for dental casts and panoramic radiographs. American Board of Orthodontics. Am J Orthod Dentofacial Orthop. 1998;114(5):589-99. DOI: 10.1016/s0889-5406(98)70179-9

5. Papageorgiou SN, Hochli D, Eliades T. Outcomes of comprehensive fixed appliance orthodontic treatment: A systematic review with meta-analysis and methodological overview. Korean J Orthod. 2017;47(6):401-13. DOl: 10.4041/kjod.2017.47.6.401

6. Chung CH, Tadlock LP, Barone N, Pangrazio-Kulbersh V, Sabott DG, Foley PF, et al. Common errors observed at the American Board of Orthodontics clinical examination. Am J Orthod Dentofacial Orthop. 2017;152(2):139-42. DOI: 10.1016/j.ajodo.2017.05.012

7. Allareddy V, Shin K, Marshall SD, Southard TE. Characteristics of an excellent orthodontic residency program. Am J Orthod Dentofacial Orthop. 2019;156(4):522-30. DOI: 10.1016/j. ajodo.2018.10.026

8. Barbosa-Lis D, Zapata O, Carvajal A, Franco C, Rodriguez S, Florez A, et al. Outcome of orthodontic treatments and its relationship to malocclusion complexity. Int J Odontostomatol. 2014;8(2):201-6. DOI: 10.4067/S0718-381X2014000200008

9. Carvajal-Florez A, Barbosa-Lis DM, Zapata-Norena OA, Marin-Velasquez JA, Afanador-Bayona SA. Orthodontic treatment outcomes obtained by application of a finishing protocol. Dental Press J Orthod. 2016;21(2):88-94. DOI: 10.1590/2177-6709.21.2.088-094.oar

10. Verma R, Utreja AK, Singh SP, Jena AK. Implementation of post treatment critical evaluation improved the quality of orthodontic care in postgraduate orthodontic clinic: A 10 years comparative study. Indian J Dent. 2015;6(3):125-9. DOI: 10.4103/0975-962X.163035

11. Pacheco-Pereira C, Pereira JR, Dick BD, Perez A, Flores-Mir C. Factors associated with patient and parent satisfaction after orthodontic treatment: a systematic review. Am J Orthod Dentofacial Orthop. 2015;148(4):652-9. DOI: 10.1016/j.ajodo.2015.04.039

12. Alfaro O, Echeverri A, Plata J. Validez y confiabiidad de un instrumento sobre satisfacción del paciente en ortodoncia [Trabajo de Grado). Bucaramanga: Universidad Santo Tomás; 2017.

13. Agudelo A, Valencia L, Oullón E, Betancur L, Restrepo L, Peláez L. Satisfacción y factores asociados en estudiantes usuarios del servicio odontológico de la institución prestadora de servicios de salud “IPS Universitaria” (Medellín). Rev Fac Odontol Univ Antioq. 2008;19(2):13-23. 
14. Torres-González G, León-Manco R. Nivel de satisfacción de los pacientes atendidos en el Servicio de Ortodoncia de una Clínica Dental Docente peruana. Rev Estomatol Herediana. 2015;25(2):122-32.

15. Dean A, Sullivan K, Soe M. OpenEpi: Open Source Epidemiologic Statistics for Public Health. 2016 [Available from: www.OpenEpi.com).

16. Fayers PM, Machin D. Multi-item Scales. Quality of Life: The Assesment Analysis and Interpretation of Patient-Reported Outcomes. Second ed. Great Britain: Willey; 2007. p. 109-30.

17. Tristán-López A. Modificación al modelo de Lawshe para el dictamen cuantitativo de la validez de contenido de un instrumento objetivo. Avances en Medición. 2008;6:37-48.

18. Landis J, Koch G. The measurement of observer agreement for categorical data. Biometrics. 1977;33(1):159-74.

19. República de Colombia, Ministerio de Salud. Resolución No. 08430 de 4 de octubre de 1993. Bogotá 1993.

20. Santiago JJ, Martinez CJ. Use of the Objective Grading System of the American Board of Orthodontics to evaluate treatment at the Orthodontic Graduate Program Clinic, University of Puerto Rico, 2007-2008. P R Health Sci J. 2012;31(1):29-34.

21. Cansunar HA, Uysal T. Relationship between pretreatment case complexity and orthodontic clinical outcomes determined by the American Board of Orthodontics criteria. Angle Orthod. 2014;84(6):974-9. DOI: 10.2319/010114-001.1

22. Song GY, Baumrind S, Zhao ZH, Ding Y, Bai YX, Wang L, et al. Validation of the American Board of Orthodontics Objective Grading System for assessing the treatment outcomes of Chinese patients. Am J Orthod Dentofacial Orthop. 2013;144(3):391-7. DOI: 10.1016/j.ajodo.2013.04.018

23. Barrera J, Meza D, Herrera C. Evaluación de los tratamientos terminados en un posgrado de ortodoncia según los parámetros ABOs 2014 [Available from: https://www.ortodoncia.ws/ publicaciones/2014/art-15/\#).

24. Campbell CL, Roberts WE, Hartsfield JK, Jr., Qi R. Treatment outcomes in a graduate orthodontic clinic for cases defined by the American Board of Orthodontics malocclusion categories. Am J Orthod Dentofacial Orthop. 2007;132(6):822-9. DOI: 10.1016/j.ajodo.2006.05.036 
18 Relación entre la calidad de los tratamientos de ortodoncia finalizados y la satisfacción en pacientes atendidos en la Universidad Santo Tomás desde 2018 hasta 2019

25. Yang-Powers LC, Sadowsky C, Rosenstein S, BeGole EA. Treatment outcome in a graduate orthodontic clinic using the American Board of Orthodontics grading system. Am J Orthod Dentofacial Orthop. 2002;122(5):451-5. DOI: 10.1067/mod.2002.128464

26. Papageorgiou SN, Höchli D, Eliades T. Outcomes of comprehensive fixed appliance orthodontic treatment: A systematic review with meta-analysis and methodological overview. Korean J Orthod. 2017;47(6):401-13. doi:10.4041/kjod.2017.47.6.401.

27. Tsichlaki A, Chin SY, Pandis N, Fleming PS. How long does treatment with fixed orthodontic appliances last? A systematic review. Am J Orthod Dentofacial Orthop. 2016;149(3):308-18. DOI: 10.1016/j.ajodo.2015.09.020 